\title{
Doença por Coronavírus de Alto Risco em 2019: Os Principais Resultados do Estudo de Coorte Multicêntrico CoronaHeart
}

\author{
High Risk Coronavirus Disease 2019: The Primary Results of \\ the CoronaHeart Multi-Center Cohort Study
}

Gilson Soares Feitosa-Filho ${ }^{1^{*}}$, Gabriella S. Sodré, Rhanniel Theodorus-Villar ${ }^{1}$

${ }^{1}$ Serviço de Cardiologia do Hospital Santa Izabel - Santa Casa de Misericórdia da Bahia; Salvador, Bahia, Brasil

Correspondence addresses: Dr. Gilson Soares Feitosa-Filho gilsonfeitosafilho@yahoo.com

Received: October 2, 2021

Revised: October 22, 2021

Accepted: December 13, 2021

Published: December 28, 2021

Data Availability Statement: All relevant data are within the paper and its Supporting Information files.

Funding: This work was the result of authors' initiative. There was no support of research or publication funds.

Competing interests: The authors have declared that no competing interests exist.

Copyright

(C) 2021 by Santa Casa

de Misericórdia da Bahia.

All rights reserved.

ISSN: 2526-5563

e-ISSN: 2764-2089

DOI: 10.35753
Background: Patients with Coronavirus Disease 2019 (COVID-19) may present high risk features during hospitalization, including cardiovascular manifestations. However, less is known about the factors that may further increase the risk of death in these patients.

Methods: We included patients with COVID-19 and high-risk features according to clinical and/or laboratory criteria at 21 sites in Brazil from June 10th to October 23rd of 2020. All variables were collected until hospital discharge or in-hospital death.

Results: A total of 2546 participants were included (mean age 65 years; $60.3 \%$ male). Overall, $70.8 \%$ were admitted to intensive care units and $54.2 \%$ had elevated troponin levels. In-hospital mortality was $41.7 \%$. An interaction among sex, age and mortality was found $(p=0.007)$. Younger women presented higher rates of death than men $(30.0 \%$ vs $22.9 \%)$, while older men presented higher rates of death than women $(57.6 \%$ vs $49.2 \%)$. The strongest factors associated with in-hospital mortality were need for mechanical ventilation (odds ratio [OR] 8.2,95\% confidence interval [CI] 5.4-12.7), elevated C-reactive protein (OR 2.3, 95\% CI 1.7-2.9), cancer (OR 1.8, $95 \% \mathrm{CI}$ 1.2-2.9), and elevated troponin levels (OR 1.8, 95\% CI 1.4-2.3). A risk score was developed for risk assessment of in-hospital mortality.

Conclusions: This cohort showed that patients with COVID-19 and high-risk features have an elevated rate of in-hospital mortality with differences according to age and sex. These results highlight unique aspects of this population and might help identifying patients who may benefit from more careful initial surveillance and potential subsequent interventional therapies.

Introdução: Pacientes doentes por Coronavírus 2019 (COVID-19) podem apresentar características de alto risco durante hospitalização, incluindo manifestações

Resumo de Artigo: Guimarães PO, de Souza FR, Lopes RD, Bittar C, Cardozo FA, Caramelli B, Calderaro D, Albuquerque CP, Drager LF, Feres F, Baracioli L, Feitosa Filho G, Barbosa RR, Ribeiro HB, Ribeiro E, Alves RJ, Soeiro A, Faillace B, Figueiredo E, Damiani LP, do Val RM, Huemer N, Nicolai LG, Hajjar LA, Abizaid A, Kalil Filho R. High risk coronavirus disease 2019: The primary results of the CoronaHeart multi-center cohort study. Int J Cardiol Heart Vasc. 2021 Oct;36:100853. doi: 10.1016/j.ijcha.2021.100853. Epub 2021 Jul 30. PMID: 34345648; PMCID: PMC8321709. 
cardiovasculares. No entanto, pouco se sabe sobre os fatores que podem aumentar ainda mais o risco de morte nesses pacientes.

Métodos: Foram incluídos pacientes com COVID-19 e características de alto risco de acordo com critérios clínicos e/ ou laboratoriais em 21 centros no Brasil de 10 de junho a 23 de outubro de 2020. Todas as variáveis foram coletadas até a alta hospitalar ou óbito hospitalar.

Resultados: Foram incluídos 2.546 participantes (idade média de 65 anos; $60,3 \%$ do sexo masculino). No geral, $70,8 \%$ foram internados em unidades de terapia intensiva e $54,2 \%$ apresentavam níveis elevados de troponina. A mortalidade hospitalar foi de $41,7 \%$. Foi encontrada interação entre sexo, idade e mortalidade $(\mathrm{p}=0,007)$. As mulheres mais jovens apresentaram maiores taxas de mortalidade do que os homens (30,0\% vs 22,9\%), enquanto os homens mais velhos apresentaram maiores taxas de mortalidade do que as mulheres (57,6\% vs 49,2\%). Os fatores mais fortemente associados à mortalidade hospitalar foram necessidade de ventilação mecânica (odds ratio [OR] 8,2, intervalo de confiança de 95\% [CI] 5,4-12,7), proteína C reativa elevada (OR 2,3, IC 95\% 1,7-2,9), câncer (OR 1,8, IC 95\% 1,2-2,9) e níveis elevados de troponina (OR 1,8, IC 95\% 1,4-2,3). Um escore de risco foi desenvolvido para avaliação de mortalidade intra-hospitalar.

Conclusões: Esta coorte mostrou que pacientes com COVID-19 e características de alto risco têm uma elevada taxa de mortalidade hospitalar com diferenças de acordo com a idade e o sexo. Esses resultados destacam aspectos únicos desta população e podem ajudar a identificar pacientes que podem se beneficiar de uma vigilância inicial mais cuidadosa e potenciais terapias intervencionistas subsequentes.

\section{COVID-19 e o Acometimento Cardiovascular}

Durante a pandemia da COVID19, em meio a incertezas das mais diversas apresentações clínicas, os pacientes com acometimento cardiovascular pareciam ter uma evolução desfavorável em relação aos outros pacientes.

Entre as apresentações clínicas, foram relatados casos de miocardite, síndrome coronariana aguda, insuficiência cardíaca, bem como elevação de troponina, considerando injúria miocárdica. No Brasil, tivemos uma taxa de mortalidade por COVID-19 de mais de 540 mil mortes até julho/2021, sendo necessária investigação dos pacientes que tiveram acometimento cardiovascular, para conhecer a evolução destes pacientes.

Fatores de risco são utilizados como preditores de gravidade e mortalidade de diversas doenças e com o COVID-19 não seria diferente. Diversos fatores de risco, tanto clínicos como laboratoriais, foram associados a prognósticos ruins em pacientes com COVID-19, especialmente aqueles relacionados ao sistema cardiovascular.

Este estudo, exclusivamente brasileiro, traznos respostas sobre o perfil de acometimento cardiovascular dos pacientes infectados pela COVID-19, além de poder estimar a probabilidade de óbito por meio de um escore diagnóstico.

\section{Desenvolvimento do Estudo CoronaHeart}

O CoronaHeart foi um estudo observacional, multicêntrico, envolvendo 21 centros de pesquisa espalhados pelo Brasil. A partir do objetivo de descrever a apresentação clínica e laboratorial de pacientes infectados por COVID-19 e identificar o perfil de pacientes que apresentam acometimento cardiovasculares para avaliar preditores de morte, também foi possível elaborar um escore de estratificação de risco cardiovascular com fins de estimar a probabilidade de óbito durante o internamento.

Foram feitas revisões em prontuários eletrônicos para selecionar os pacientes internados com COVID-19, que incluíam os pacientes positivos ou com critérios clínicos sugestivos de infecção por COVID-19, mesmo que os exames estivessem indisponíveis ou negativos, mas que apresentassem qualquer tipo de acometimento cardiovascular, identificado pela presença de marcadores laboratoriais, seja elevação de troponina ou D-dímero ou exames de imagem comprovando algum tipo de injúria cardíaca nas primeiras $48 \mathrm{~h}$ de internamento hospitalar.

Observamos nas apresentações clínicas que poucos pacientes apresentaram arritmias cardíacas e, aqueles que apresentaram, tiveram bom desfecho ao final. Aqueles pacientes com 
síndrome coronariana aguda apresentavam, em sua maioria, ao cateterismo, grande presença de trombos nas coronárias.

\section{Métodos}

$\mathrm{Na}$ análise estatística, as variáveis categóricas foram expressas em porcentagem e as variáveis contínuas foram expressas como média, desviopadrão ou mediana (intervalo interquartil). A coorte foi descrita comparando pacientes do sexo masculino e feminino. Seus perfis foram comparados usando testes de qui-quadrado para variáveis categóricas e testes de Mann-Whitney para a maioria das variáveis contínuas. Foram incluídas $2.496(98,1 \%)$ características dos pacientes e os dados ausentes foram imputados por meio de equações encadeadas usando o package mice. A interação entre sexo e idade na mortalidade foi identificada nas curvas de Kaplan-Meier e gráficos de barras de acordo com os quartis de idade. A análise multivariada de regressão logística para mortalidade intrahospitalar foi apresentada considerando fatores de base e achados laboratoriais. Como nem todos os testes laboratoriais de linha de base estavam disponíveis em toda a população, a análise multivariável foi realizada com uma subamostra de 1.323 pacientes. Um nomograma foi formulado com base nos resultados do modelo final usando o pacote rms. Todas as análises foram feitas com o software R 4.0.2 (R Core Team, Vienna, Austria, 2020).

\section{Desfehos Clínicos (Figura 1)}

O tempo de hospitalização foi em média de 14 dias (8-24). Entre estes, 70,8\% necessitaram de leito de UTI, permanecendo em média 6 dias (0-14). Dos que estiveram na UTI, 45,9\% necessitaram de ventilação mecânica.

Os homens tiveram maior taxa de admissão na UTI do que as mulheres (73,3\% vs 67,0\%). A Figura 1 apresenta as curvas para mortalidade hospitalar distribuídas por idade e sexo.

Por meio da análise univariável, entre os preditores de mortalidade, observamos os valores de troponina acima de 2,17 vezes acima do limite da normalidade, D-dímero 4,34 vezes acima do limite da normalidade e 22,7 vezes acima do limite da normalidade para Proteína C Reativa.

Figura 1. Taxa de mortalidade hospitalar por idade e sexo.

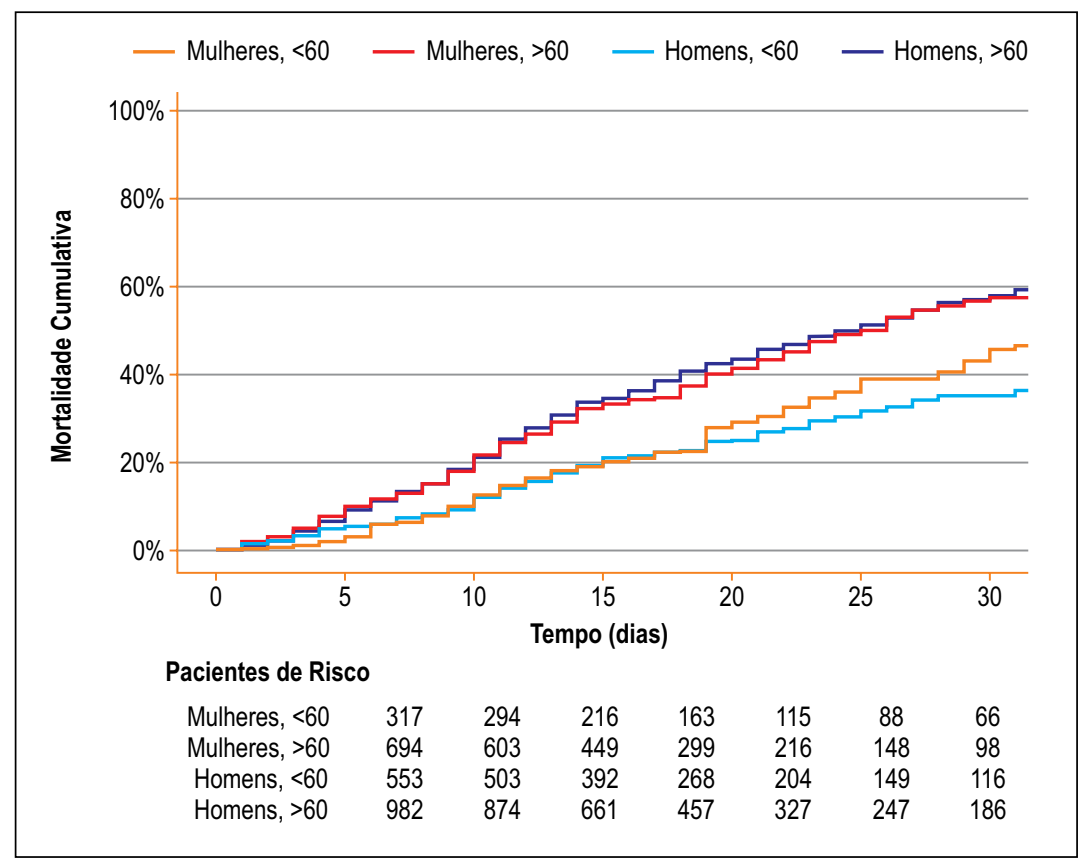


Através da análise multivariável, o aumento da idade, necessidade de suporte de oxigênio na admissão, passado de neoplasia ou neoplasia ativa, presença de mialgia na admissão, bem como a elevação de D-dímero acima de 4,4 vezes do limite da normalidade, troponina 2 vezes acima do limite da normalidade, PCR 20 vezes acima dos valores de referência e plaquetopenia foram associados com maior mortalidade intra-hospitalar.

\section{Aplicabilidade Clínica do Escore CoronaHeart (Figura 2)}

Apartir desteestudo, conseguimos obter informações sobre o tipo de pacientes que são acometidos pela COVID-19 e os principais tipos de alterações cardiológicas que são encontradas. Desta maneira, podese compreender o comportamento fisiopatológico da doença, bem como seu aspecto evolutivo.

Figura 2. O escore de risco do CoronaHeart.

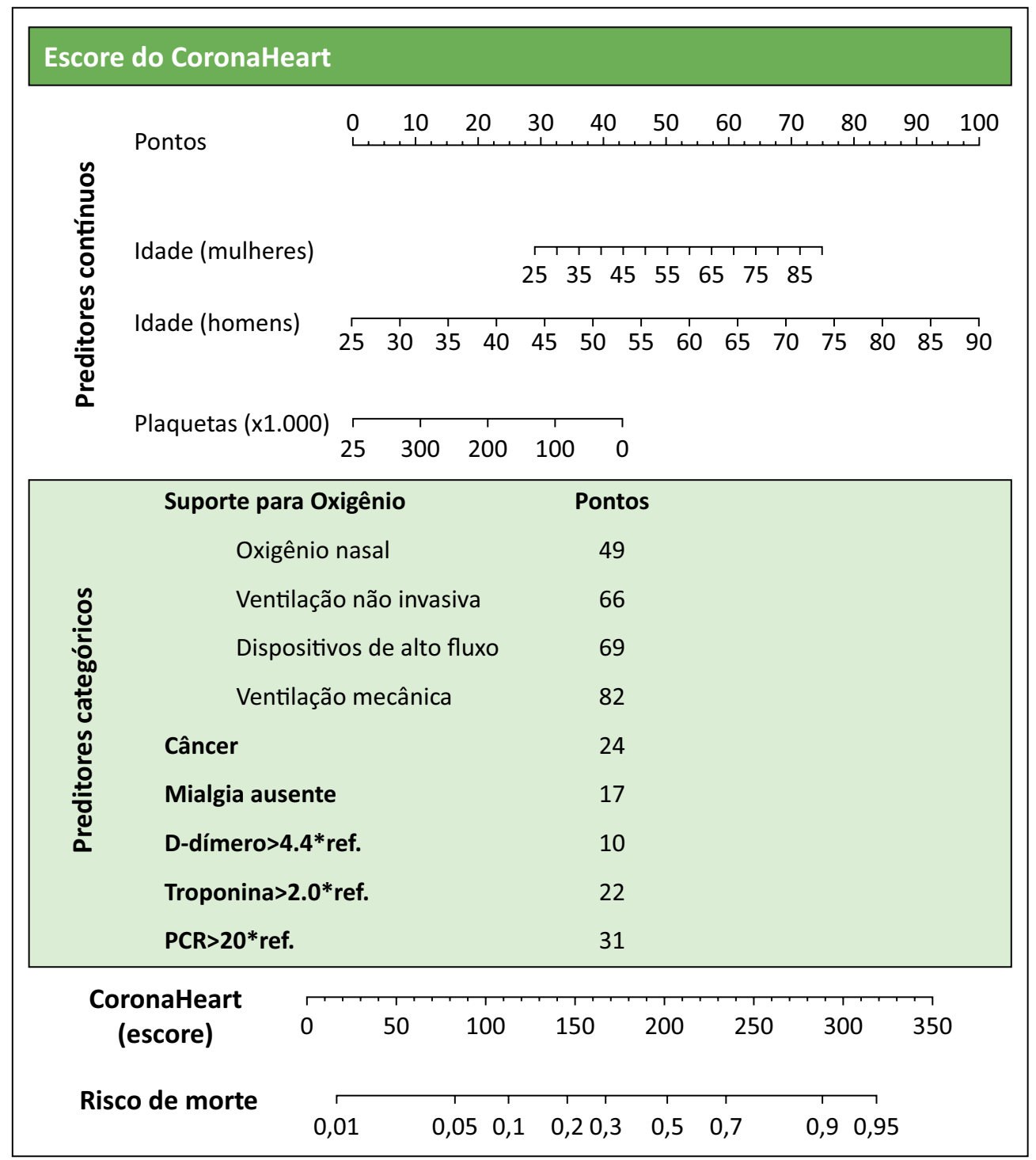

PCR: proteína C reatiba. Ref.: valor de referência. 
Por meio de um escore de prognóstico, torna-se possível estimar a probabilidade de complicações cardiovasculares durante o internamento de um paciente infectado pelo novo coronavírus.

O CoronaHeart Risk Score foi construído com base nos coeficientes do modelo logístico. Um nomograma foi desenvolvido para ajudar os médicos a calcularem a probabilidade de que um paciente com COVID-19 de alto risco tenha um desfecho desfavorável durante o internamento hospitalar.

\section{Participação do Hospital Santa Izabel}

O registro CoronaHeart é uma das maiores coortes do mundo sobre o acometimento por COVID-19 em pacientes cardiopatas ou com critérios de risco cardiovascular. Dos vários centros participantes do país, o Hospital Santa Izabel foi o $3^{\circ}$ centro a contribuir com maior número de pacientes e, com isso, muitos dos dados apresentados são fruto de importante contribuição baiana. Isso é importante, não só para projetar o Centro de Pesquisa em Cardiologia do Hospital Santa Izabel como um dos principais centros de pesquisa do país, mas também por trazer representatividade da população baiana aos resultados encontrados.

\section{Referência}

1. Guimarães PO, de Souza FR, Lopes RD, Bittar C, Cardozo FA, Caramelli B, Calderaro D, Albuquerque CP, Drager LF, Feres F, Baracioli L, Feitosa Filho G, Barbosa RR, Ribeiro HB, Ribeiro E, Alves RJ, Soeiro A, Faillace B, Figueiredo E, Damiani LP, do Val RM, Huemer N, Nicolai LG, Hajjar LA, Abizaid A, Kalil Filho R. High risk coronavirus disease 2019: The primary results of the CoronaHeart multi-center cohort study. Int J Cardiol Heart Vasc. 2021 Oct;36:100853. doi: 10.1016/j.ijcha.2021.100853. Epub 2021 Jul 30. PMID: 34345648; PMCID: PMC8321709. 